PASJ: Publ. Astron. Soc. Japan , 1-??,

(C) 2018. Astronomical Society of Japan.

\title{
Discovery of a new dwarf nova, TSS J022216.4+412259.9: WZ Sge-type dwarf novae breaking the shortest superhump period record
}

\author{
Akira Imada, ${ }^{1}$ Kaori Kubota, ${ }^{1}$ Taichi Kato, ${ }^{1}$ Daisaku Nogami, ${ }^{2}$ Hiroyuki Maehara, ${ }^{3}$ \\ Kazuhiro Nakajima, ${ }^{4}$ Makoto Uemura, ${ }^{5}$ and Ryoko Ishioka ${ }^{6}$ \\ ${ }^{1}$ Department of Astronomy,Faculty of Science, Kyoto University, Sakyo-ku, Kyoto 606-8502 \\ ${ }^{2}$ Hida Observatory, Kyoto University, Kamitakara, Gifu 506-1314 \\ ${ }^{3}$ VSOLJ, Namiki 1-13-4, Kawaguchi, Saitama 332-0034, Japan \\ ${ }^{4}$ VSOLJ, 124 Isatotyo Teradani, Kumano, Mie, Japan \\ ${ }^{5}$ Hiroshima Astrophysical Science Center, Hiroshima University, Hiroshima 739-8526, Japan \\ ${ }^{6}$ Subaru Telescope, National Astronomical Observatory of Japan 650 North A'ohoku Place, Hilo, \\ HI 96720, U.S.A. \\ a_imada@kusastro.kyoto-u.ac.jp
}

(Received ; accepted )

\begin{abstract}
We report on the time-resolved CCD photometry of a newly discovered variable star, TSS J022216.4+412259.9 during the outburst in 2005 November-December brightening. The obtained light curves unambiguously showed 0.2-0.3 mag modulations, which we confirmed to be the superhump observed among SU UMa-type dwarf novae. We also performed a period search for the data obtained during the outburst plateau phase, and revealed the existence of the two periodicities: $0.054868(98)$ days for the first two nights and 0.055544(26) days for the following plateau phase. This bi-periodicity is hardly observed in usual SU UMa-type dwarf novae, but characteristic of WZ Sge-type stars. We undoubtedly detected a rebrightening in the post-outburst stage, which is typical of short-period SU UMa-type dwarf novae including WZ Sge-type stars. These observations suggests that TSS J022216.4+412259.9 may be a new WZ Sge stars breaking the shortest superhump period of 0.05648 days for V592 Her among this class with a known superhump period so far.

Key words: accretion, accretion disks — stars: dwarf novae — stars: individual (TSS J022216.4+412259.9) — stars: novae, cataclysmic variables — stars: oscillations
\end{abstract}

\section{Introduction}

Since the advent of the high speed CCD photometry, many studies have been performed concerning the variability of dwarf novae (Warner 1995; Kato et al. 2004), which have led a significant progress in understanding many aspects of dwarf novae-outburst. WZ Sge stars, an extreme subclass of SU UMa-type dwarf novae, show many peculiarities both in outburst and quiescence. One of the enigmatic phenomenon is that they exhibit so called "early superhumps" on the early stage of the superoutburst of WZ Sge stars (Kato et al. 1996; Osaki, Meyer 2002; Kato 2002; Patterson et al. 2002). The period of the humps is almost identical to that of the orbital period of the system. Another peculiarity is that most of WZ Sge stars show rebrightenings (Kuulkers et al. 1996; Patterson et al. 1998) after the main outburst. Extensive monitoring of WZ Sge stars, as well as insightful theoretical works, have shed some light on elucidating these origin and revealing the diversity of them, while less than ten objects have been certified as this class. However, interpretation of the aforementioned phenomena is controversial.
Recently, there has arisen a new problem regarding the evolution of dwarf novae. According to a population synthesis, there should be more dwarf novae below the period gap than observed (Howell et al. 1997). Howell et al. (1997) further suggested that almost all of CVs should have the orbital period below the period gap. However, observations indicate that the number of CVs above the period gap is comparable to that of CVs below the period gap (Gänsicke 2005). Another calculation suggests the "theoretical period minimum" of dwarf novae will lie as short as $64 \mathrm{~min}$ (Kolb, Baraffe 1999) under the secular evolution. Willems et al. (2005), on the other hand, has shown that the "period minimum" becomes around $80 \mathrm{~min}$ by an inclusion of an effect of a circumbinary disk. After reaching the "period minimum", the system comprises a degenerate secondary, which gives rise to inversion of the mass-radius relation. Then the orbital period of the system will become longer as the CV evolves. Meanwhile, well accepted is that the "obser- 
vational period minimum" lies around 78 min. ${ }^{12}$ As far as the evolutional scenario of dwarf novae is concerned, intensive studies should be awaited especially from the theoretical side (see Howell et al. (2001) for a review.).

TSS J022216.4+412259.9 was firstly discovered by Quimby et al. (2005) as a candidate of an ongoing supernova on 2005 November 16.1 (UT) with about $15.5 \mathrm{mag}$. The object maintained almost the same magnitude until 2005 November 17.1 (UT), which was confirmed by 45 cm ROTSE-IIIb telescope at the McDonald Observatory. Negative observations were carried out at the same site, giving $16.5 \mathrm{mag}$ as an upper limit on 2005 November 15.1 (UT). On 2005 November 18, an optical spectrum was obtained with a $2.2 \mathrm{~m}$ telescope located on the University of Hawaii, which revealed the dwarf nova nature of the eruptive object by unambiguous detection of $\mathrm{H} \alpha$ and HeII 4686 Ain emission, as well as a blue continuum (Quimby et al. 2005). There is no optical counterpart in USNO B1.0 and 2MASS catalog within the 5 arcsec circle. This suggests that the quiescent magnitude of the object is fainter than 21 mag.

In this letter, we report on the results of CCD photometric observations during the 2005 November long outburst of TSS $022216.4+412259.9$ (hereafter, aliased TSS J0222), mainly focusing on detection of two periodicities during the outburst plateau stage. Detailed discussion will be published in the forthcoming paper.

\section{Observations}

Time-resolved CCD photometry was carried out from 2005 November 21 to 2005 December 14 at 4 sites using 25-60 cm telescopes. More information about each site is described in table 1 . The total data points exceeded 10000. Typical exposure time was 10-30 sec with no filter. The resultant band pass is close to $R_{c}$-band. The differential magnitude among each sites was adjusted to that of the Kyoto site. We used USNO-A2.0 1275-01425512 ( $B=12.8, R=12.6$ ) as a comparison star, whose constancy was checked by several stars in the same image. Figure 1 illustrates a finding chart of TSS J0222. Kyoto team used java-based PSF photometry package developed by one of authors (TK). Hida and Saitama used IRAF DAOPHOT and APPHOT. Data of Mie were analysed by FitsPhot4.1. ${ }^{3}$ Because of the faintness of TSS J0222, accurate calibration of the magnitude was prevented on some nights. However, such uncertainty would not influence on the following analysis in which we mainly focus on the periodicity of photometric variations. Heliocentric correction is also made for our whole run.

1 V485 Cen and EI Psc, recently discovered dwarf novae with the periods breaking the minimum, are suggested to have experienced another evolution with an evolved secondary (Podsiadlowski et al. 2003).

2 Theoretical works suggest the value of the period minimum depends on chemical abundance of the secondary star.

3 http://www.geocities.jp/nagai_kazuo/dload-1.html

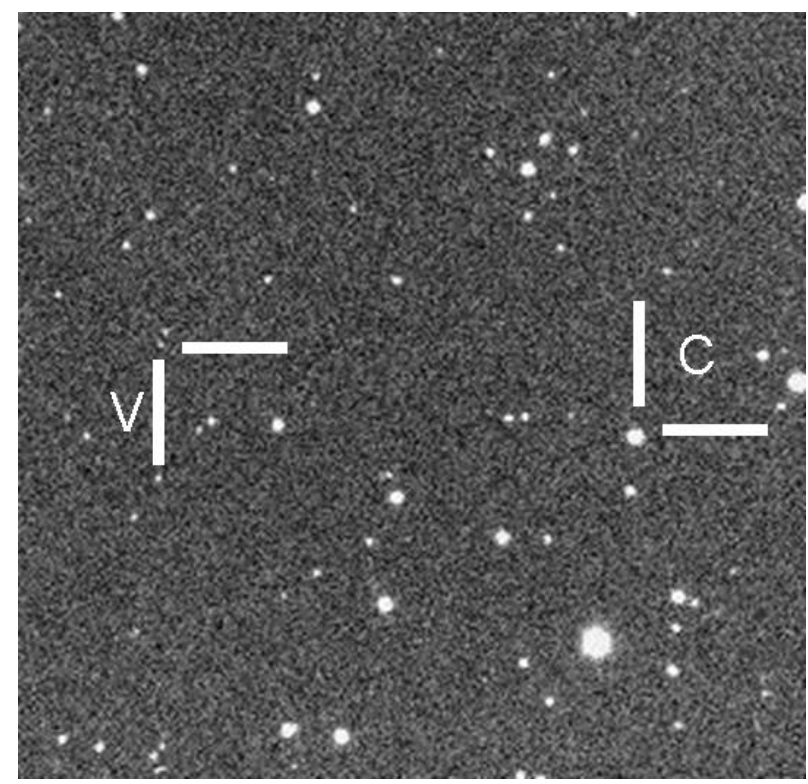

Fig. 1. Finding chart of TSS J0222 from our original data. The field of view is about $16^{\prime} \times 8^{\prime}$. The north is up and the east is to the left. The variable and the comparison star are denoted by $V$ and $C$, respectively.

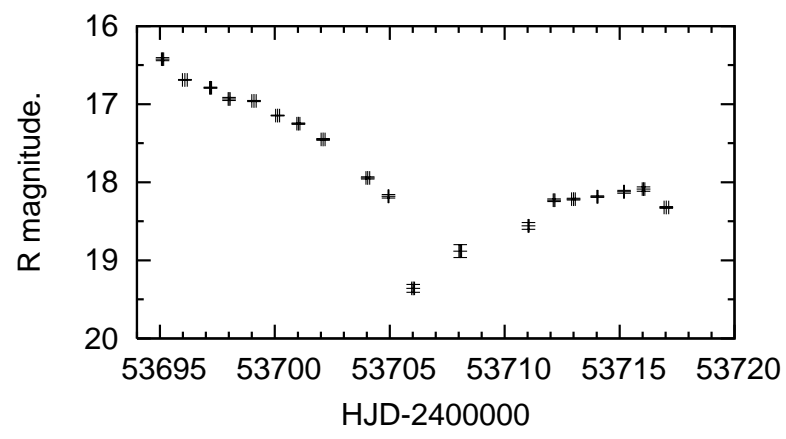

Fig. 2. The daily averaged light curve during the outburst. The vertical and horizontal axes denote $R$ magnitude and HJD, respectively. The magnitude of the comparison is $R=$ 12.6. The error bar denotes standard error. Due to the faintness of TSS J0222, the accuracy of the magnitude calibration was dependent on the weather condition so that we excluded a few data. Nevertheless, we unambiguously detected the rebrightenings around HJD 2453708. 
Table 1. Information about each site.

\begin{tabular}{lccc}
\hline \hline Site & Telescope & $\operatorname{Exp}(\mathrm{s})^{\dagger}$ & $\mathrm{N}($ days $)$ \\
\hline \hline Kyoto, Japan & $40 \mathrm{~cm}$ & 30 & 20 \\
Hida, Japan & $60 \mathrm{~cm}$ & 10 & 1 \\
Saitama, Japan & $25 \mathrm{~cm}$ & 30 & 2 \\
Mie, Japan & $25 \mathrm{~cm}$ & 30 & 1 \\
\hline$\dagger$ Exposure time. & & \\
$\ddagger$ Number of observed nights.
\end{tabular}

\section{Results and discussion}

\section{1. light curves}

Figure 2 displays the overall light curve of this outburst. At the onset of our observations, TSS J0222 was at the magnitude of 16.4. After that the magnitude linearly faded down with the rate of $0.1 \mathrm{mag} \mathrm{d}^{-1}$. Some WZ Sge stars show a rapid decline just after the maximum brightness with $\sim 0.5 \mathrm{mag} \mathrm{d}^{-1}$, presumably due to viscous decay in the accretion disk (Kato et al. 2002). In the case of TSS J0222, we cannot specify such a rapid declining took place due to the lack of the our run at the early stage of the superoutburst. After the end of the main plateau stage, TSS J0222 faded down as faint as our detection limit ( $19.5 \mathrm{mag}$ ) on HJD 2453706. However, we detected a rebrightening on HJD 2453708 at the magnitude of 18.8. Judging from figure 2, the rebrightening lasted at least 9 days. Because of an intrinsic faintness of TSS J0222, we could not specify the overall feature of the rebrightenings. Phenomenologically, three types of rebrightenings may exist. AL Com and WZ Sge itself kept bright for a week or even more, although about 1 mag-fluctuations were observed with a time scale of a few days in WZ Sge. The overall light curves of rebrightenings are not discrete, but somewhat long-lasting. Here we call this type of rebrighenings "type-A". On the other hand, regular rebrightenings were seen in EG Cnc. Here we call this kind of rebrightenings "type-B". Finally, not only WZ Sge stars but also some SU UMa stars show a short rebrightening. Such systems include RZ Leo (Ishioka et al. 2001). We call "type-C" for the rebrightening. The observed rebrightening of TSS J0222 apparently belongs to the "type-A" category. The reason why different rebrightenings emerge even in the same system, is not known (for a relevant paper, see e.g., Kuulkers et al. 1996; Osaki et al. 2001).footnoteFor example, WZ Sge shows two types of rebrightenings. In 1978 and 2001 superoutburst, the object exhibited the type-A rebrightening, while no rebrightening was observed in 1946 superoutbutst (Patterson et al. 1981; Ishioka et al. 2002.

Figure 3 displays enlarged light curves on HJD 2453696 and HJD 2453697. There is almost no feature on HJD 2453696 at a glance. However, double-peaked features can be seen when the data of HJD 2453695 and HJD 2453696 are folded with 0.054868 days (discussed later). As for the light curve on HJD 2453697, we can clearly see a rapid-rise and a slow decline feature, which is typical for the superhump profile. We suggest that some kind of transition

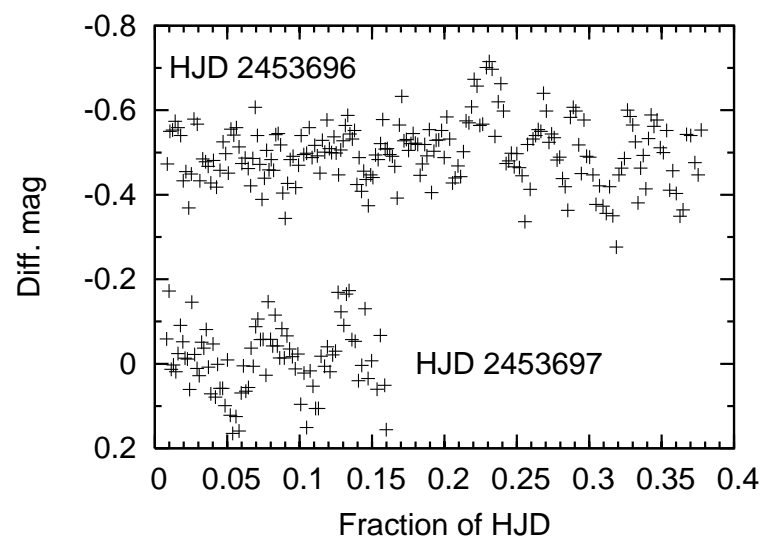

Fig. 3. Light curves on HJD 2453696 and HJD 2453697, second and third night of our observations. Both light curves were subtracted a linear declining trend. Note that there are vague features on HJD 2453696, while there are features of superhumps on HJD 2453697.

took place between HJD 2453696 and HJD 2453697, and that the feature we observed on HJD 2453697 was genuine superhumps, as is observed in SU UMa stars during superoutbursts. This kind of transition has been observed in WZ Sge stars amid the plateau stage (e.g., Nogami et al. 1997; Patterson et al. 1998; Ishioka et al. 2002). Therefore, we can reasonably conclude that we did observe early superhumps on HJD 2453695 and HJD 2453696, and genuine superhumps from HJD 2453697 on, respectively.

\section{2. period analysis}

The results of period analyses during the plateau phase are represented in figure 4. After subtracting a linear decline trend for each run, we performed the phase dispersion minimization method (PDM, Stellingwerf (1978)) for the data obtained on HJD 2453695 and HJD 2453696, corresponding to the early superhump phase (upper panel), and for following 7 days, corresponding to the genuine superhump phase (lower panel), respectively. The statistical errors were estimated by using Lafler-Kinman class method (Fernie 1989). As can be obviously seen in figure 4, two different periodicities were exhibited: $0.054868(98)$ days for early superhumps, and 0.055544(26) days for genuine superhumps, respectively. The statistical errors were estimated by using Lafler-Kinman class method (Fernie 1989). The statistical F-tests yielded significance levels of the signals $74 \%$ for early superhumps and $83 \%$ for genuine superhumps, respectively. Note that the resultant superhump period is the shortest record among WZ Sge stars, exceeding 0.05648(2) days of V592 Her (Kato et al. 2002). Figure 5 shows averaged light curves during these stages folded by the periods obtained above. Note that the profile of the upper panel is not singly peaked, but definitely doubly peaked, similar to that observed in the early phase of WZ Sge and AL Com (Ishioka et al. 2002). On the other hand, the lower panel illustrates a singly peaked profile. The rapid rise and slow decline of the profile are quite typical for superhumps of SU UMa stars. 

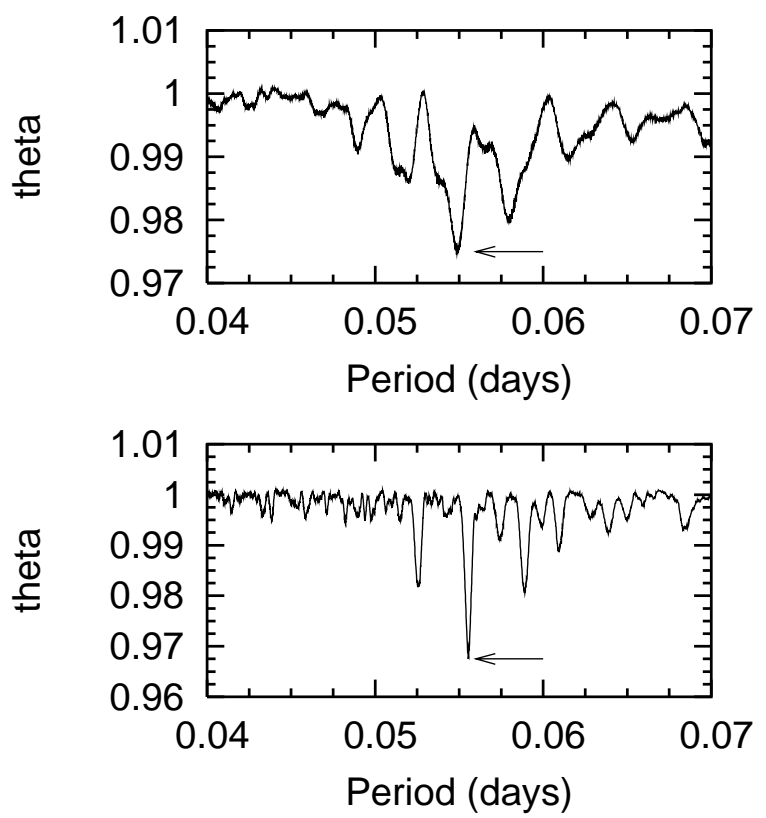

Fig. 4. Theta diagrams of the period analyses using the PDM method for the data obtained during the first two days (Top panel) and the following nights of the plateau stage (Bottom panel). For the first two days, 0.054868(98) days is the best estimated period, while the periodicity of $0.055544(26)$ days is shown since HJD 2453697. We interpreted the latter as the mean superhump period of TSS J0222.
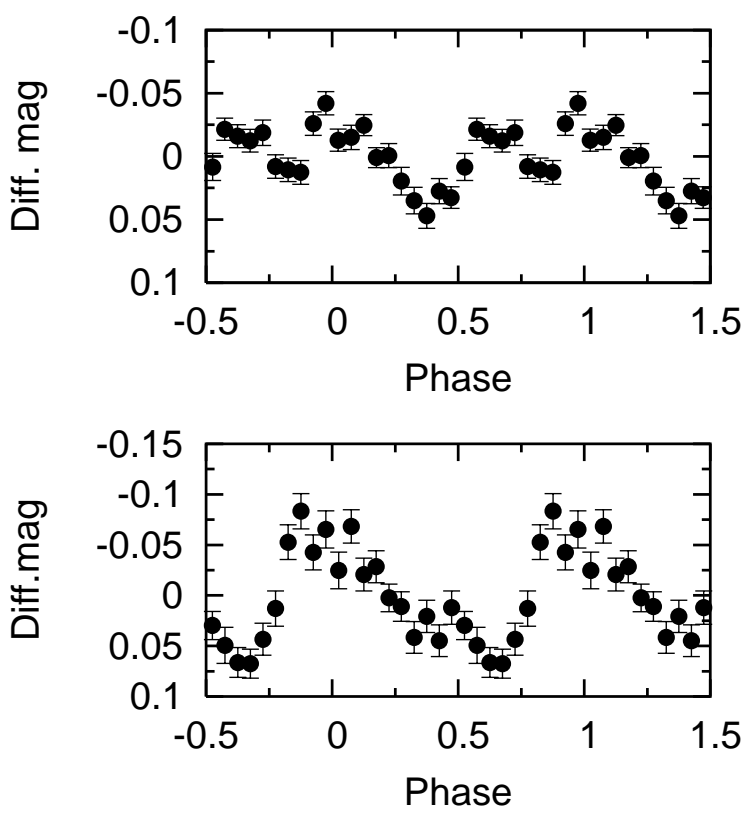

Fig. 5. Phase-averaged light curves during the two stages folded with the above mentioned period, 0.054868 days and 0.055544 days, respectively. One can see double-peaked profiles, as is observed in WZ Sge-type dwarf novae in the top panel. On the other hand, a rapid rise and slow decline, characteristic of superhumps, is evident on the bottom panel.

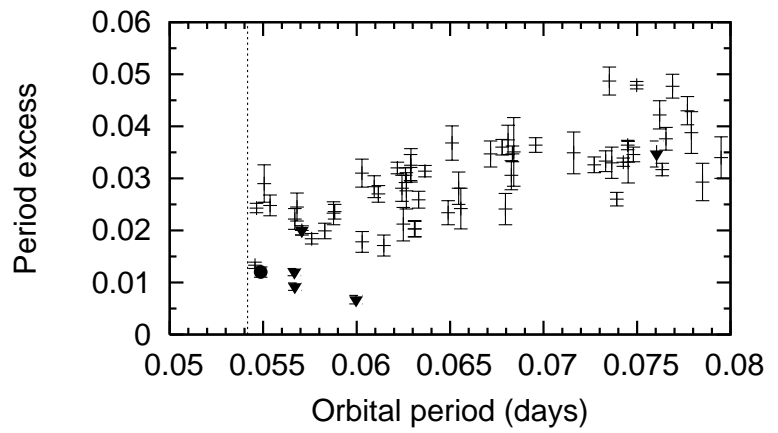

Fig. 6. $\epsilon$-period diagram of SU UMa-type dwarf novae. TSS J0222 and confirmed WZ Sge stars are also included with the filled circle and filled triangles, respectively. The figure is drawn based on table 9 of Patterson et al. (2005). The vertical dotted line indicates the observed period minimum of SU UMa stars on the secular evolution path.

\subsection{TSS J0222 as a WZ Sge star}

The present superoutburst of TSS J0222 showed two types of humps, a rebrightening, and a large-amplitude of the eruption larger than $6 \mathrm{mag}$, all of which are characteristic of WZ Sge stars (Howell et al. 1995; Imada et al. 2005). Judging from the results mentioned before, we can reasonably conclude that TSS J0222 is a new member of WZ Sge stars with the shortest period ever known. We further investigated the mass ratio of the object based on an empirical relation derived by Patterson (1998) as follows:

$$
\epsilon=\frac{0.23 q}{1+0.27 q},
$$

where $\epsilon$ and $q$ are the fractional superhump excess and mass ratio, respectively. Because the radial velocity study has not been performed for TSS J0222, the orbital period of TSS J0222 cannot be exactly specified. However, taking into account the fact that the period of the early superhump is almost the same as that of the orbital period (Patterson et al. 1998; Patterson et al. 2002; Ishioka et al. 2003), we can validly use the early superhump period above obtained (0.054868 days) as the orbital period of TSS J0222. Thus the fractional superhump excess is estimated as being $0.0123(23)$ with a little algebra. Substituting this value into the equation (1), we obtained $\mathrm{q}=0.054(10)$. The value is quite a similar to that listed in Patterson et al. (2005) for some WZ Sge stars. This implies that the mass of the secondary star might be as low as $0.075 M_{\odot}$ even if the primary white dwarf reaches the Chandrasekhar mass.

Figure 6 shows $\epsilon-P_{\text {orb }}$ diagram recently refined by Patterson et al. (2005), together with the data of TSS J0222 derived above. Note that the location of TSS J0222 is close to that of WZ Sge and AL Com, rather than that of SU UMa-type dwarf novae with short orbital periods, e.g., WX Cet and SW UMa. This suggests that TSS J0222 bears some resemblance to WZ Sge and AL Com. 


\section{Summary}

In this letter, we reported on the newly discovered dwarf nova, TSS J0222. The obtained light curves provided enough evidence for early superhumps and genuine superhumps, characteristic of WZ Sge-type dwarf novae. We derived the superhump period was $0.055544(26)$ days, as well as the early superhump period of $0.054868(98)$ days. The value is the shortest period among WZ Sge stars. Although a faintness of the object prevented an accurate calibration of magnitude after the post-plateau stage, we detected a long rebrightening during this stage, indicative of the WZ Sge nature of the system. The overall rebrightenings was classified into "type-A" in the letter, similar to those observed in the 1995 superoutburst of AL Com. Assuming that the early superhump period is exactly the same as the orbital period, we roughly derived the mass ratio of TSS J0222 to be 0.054(10). This may indicate the mass of the secondary star is low, like WZ Sge and AL Com. In the future, further observations with a large telescope should be performed to accurately determine the orbital period and the mass ratio of the system.

We would express our gratitude to VSNET observers. This work is supported by Grants-in-Aid for the 21st Century COE "Center for Diversity and Universality in Physics" from the Ministry of Education, Culture, Sports, Science and Technology (MEXT), and also by Grantsin-Aid from MEXT (No. 13640239, 16340057, 16740121, 17740105).

\section{References}

Fernie, J. D. 1989, PASP, 101, 225

Gänsicke, B. T. 2005, in ASP Conf. Ser. 330, The astrophysics of Cataclysmic Variables and Related Objects, ed. J. M. Hameury, \& J. M. Lasota (San Francisco: ASP), 3

Howell, S. B., Nelson, L. A., \& Rappaport, S. 2001, ApJ, 550, 897

Howell, S. B., Rappaport, S., \& Politano, M. 1997, MNRAS, 287,929

Howell, S. B., Szkody, P., \& Cannizzo, J. K. 1995, ApJ, 439, 337

Imada, A., et al. 2005, PASJ, 57, 193

Ishioka, R., et al. 2001, PASJ, 53, 905

Ishioka, R., et al. 2003, PASJ, 55, 683

Ishioka, R., et al. 2002, A\&A, 381, L41

Kato, T. 2002, PASJ, 54, L11

Kato, T., Nogami, D., Baba, H., Matsumoto, K., Arimoto, J., Tanabe, K., \& Ishikawa, K. 1996, PASJ, 48, L21

Kato, T., Uemura, M., Ishioka, R., Nogami, D., Kunjaya, C., Baba, H., \& Yamaoka, H. 2004, PASJ, 56S, 1

Kato, T., Uemura, M., Matsumoto, K., Kinnunen, T. Garradd, G., Masi, G., \& Yamaoka, H. 2002, PASJ, 54, 999

Kolb, U., \& Baraffe, I. 1999, MNRAS, 309, 1034

Kuulkers, E., Howell, S. B., \& van Paradijs, J. 1996, ApJL, 462, L87

Nogami, D., Kato, T., Baba, H., Matsumoto, K., Arimoto, J., Tanabe, K., \& Ishikawa, K. 1997, ApJ, 490, 840

Osaki, Y., \& Meyer, F. 2002, A\&A, 383, 574
Osaki, Y., Meyer, F., \& Meyer-Hofmeister, E. 2001, A\&A, 370, 488

Patterson, J. 1998, PASP, 110, 1132

Patterson, J., et al. 2005, PASP, 117, 1204

Patterson, J., et al. 1998, PASP, 110, 1290

Patterson, J., McGraw, J. T., Coleman, L., \& Africano, J. L. 1981, ApJ, 248, 1067

Patterson, J., et al. 2002, PASP, 114, 721

Podsiadlowski, Ph., Han, Z., \& Rappaport, S. 2003, MNRAS, 340, 1214

Quimby, R., et al. 2005, ATEL, 658

Stellingwerf, R. F. 1978, ApJ, 224, 953

Warner, B. 1995, Cataclysmic Variable Stars (Cambridge: Cambridge University Press)

Willems, B., Kolb, U., Sandquist, E.L., Taam, R. E., \& Dubus, G. 2005, ApJ, 635, 1263 